\title{
Влияние одноосной упругой деформации на вольт-амперную характеристику поверхностно-барьерных диодов $\mathrm{Sb}-p-\mathrm{Si}\langle\mathrm{Mn}\rangle-\mathrm{Au}$
}

\author{
(C) О.О. Маматкаримов ${ }^{1}$, О. Химматкулов ${ }^{2}$, И.Г. Турсунов ${ }^{3,4}$ \\ ${ }^{1}$ Наманганский инженерно-технологический институт, \\ 160115 Наманган, Узбекистан \\ ${ }^{2}$ Ташкентский государственный технический университет, \\ 100174 Ташкент, Узбекистан \\ 3 Чирчикский государственный педагогический институт, \\ 100700 Чирчик, Узбекистан \\ ${ }^{4}$ Национальный университет Узбекистана, \\ 100174 Ташкент, Узбекистан \\ E-mail: ikromjon0804@gmail.com
}

Поступила в Редакцию 31 октября 2019 г. В окончательной редакции 9 декабря 2019 г. Принята к публикации 11 декабря 2019 г.

\begin{abstract}
Исследовано влияние одноосной упругой деформации на вольт-амперную характеристику в поверхностнобарьерных диодах типа $\mathrm{Sb}-p$ - $\mathrm{Si}\langle\mathrm{Mn}\rangle-\mathrm{Au}$. Показано, что чувствительность обратного тока структуры к одноосному сжатию значительно превосходит соответствующую чувствительность прямого тока при одинаковых значениях приложенного напряжения. Увеличение прямого тока данных структур при деформации обусловлено внутренним усилением, связанным с перераспределением приложенного напряжения между базой и барьером.
\end{abstract}

Ключевые слова: кремний, поверхностно-барьерный диод, одноосная деформация.

DOI: 10.21883/FTP.2020.05.49263.9303

\section{1. Введение}

Известно [1], что тензочувствительность поверхностно-барьерных диодов (ПБД) $\mathrm{Sb}-p-\mathrm{Si}\langle\mathrm{Mn}\rangle-\mathrm{Au}$ при статическом воздействии всестороннего гидростатического сжатия значительно превосходит тензочувствительности базовых компенсированных кристаллов кремния. Если при всестороннем гидростатическом сжатии (ВГС) в кремнии расщепления энергетических зон не происходит и все долины смещаются одинаково, то при одноосном сжатии в зависимости от ориентации оси деформации относительно кристаллографического направления энергетические зоны расщепляются соответствующим образом и энергетические долины смещаются по-разному. Поэтому естественно ожидать, что тензоэффект в структурах ПБД при ориентированной деформации должен отличаться от тензоэффекта, возникающего при ВГС.

Цель настоящей работы - изучение вольт-амперной характеристики поверхностно-барьерных диодов $\mathrm{Sb}-p-\mathrm{Si}\langle\mathrm{Mn}\rangle-\mathrm{Au}$ при одноосной упругой деформации.

\section{2. Методика эксперимента}

Нами исследовано влияние одноосной упругой деформации (ОУД) в статическом режиме воздействия на вольт-амперную характеристику (BAX) ПБД типа $\mathrm{Sb}-p-\mathrm{Si}\langle\mathrm{Mn}\rangle-\mathrm{Au}$, в которых в качестве базы использована компенсированная Мn кремниевая пластина ды- рочного типа проводимости с удельным сопротивлением 300 Ом · см. Диодные структуры были изготовлены напылением золота и сурьмы на противоположные грани пластины с большой поверхностью, перпендикулярной кристаллографической оси [111]. Выпрямляющий контакт создавался на границе раздела $\mathrm{Sb}-p-\mathrm{Si}\langle\mathrm{Mn}\rangle$. Одноосное сжатие осуществляли с помощью специальной установки ОУД с пневмоусилителем [2], которая позволяет одновременно исследовать эффекты тензосопротивления и Холла при разных условиях сжатия. Исследования проводились в интервале давлений $X=0-9 \cdot 10^{8}$ Па и при температуре $T=300 \mathrm{~K}$.

\section{3. Результаты и их обсуждение}

На рис. 1 приведены относительные изменения прямого тока данных диодных структур в диапазоне одноосного сжатия $X=0-9 \cdot 10^{8}$ Па при напряжениях смещения $U=0.5,1,3 \mathrm{~B}$ (кривые $1-3$ соответственно). Видно, что прямой ток $J(X)$ с ростом одноосного сжатия $X$ сначала монотонно возрастает, а начиная с $X>6 \cdot 10^{8}$ Па, в зависимостях $J(X) / J(0)$ наступает участок замедления и насыщения, при этом максимальное изменение прямого тока при $U=2$ В составляет 2.5 раза. Следует отметить, что токовая чувствительность при прямом смещении $U>2$ В не зависит от приложенного напряжения.

Проведенные измерения в обратно смещенных диодах в области напряжений, далеких от напряжения пробоя, 


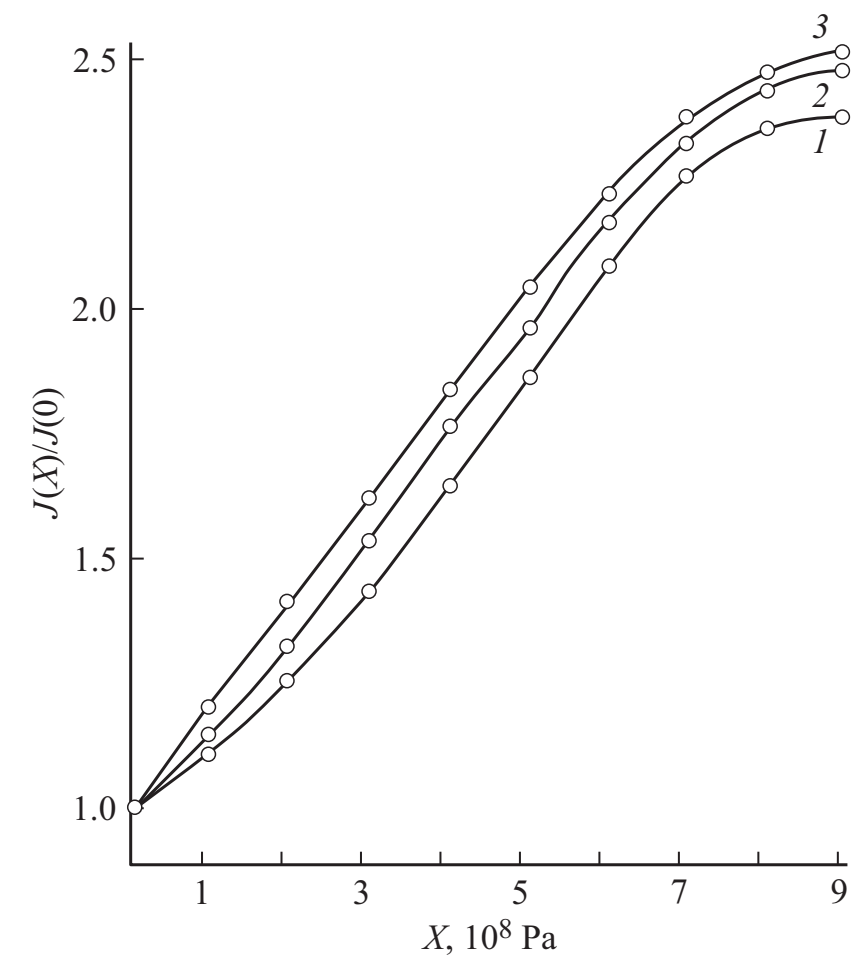

Рис. 1. Зависимости относительного изменения тока прямой ветви ВАХ ПБД $\mathrm{Sb}-p$ - $\mathrm{Si}\langle\mathrm{B}, \mathrm{Mn}\rangle-\mathrm{Au}$ от величины одноосного сжатия. $U=0.5(1), 1$ (2), $2 \mathrm{~B}(3)$.

показали, что чувствительность обратного тока к одноосному сжатию значительно превосходит соответствующую чувствительность прямого тока при одинаковых значениях приложенного напряжения.

На рис. 2 приведены зависимости относительного обратного тока данного диода от $X$ в диапазоне сжатий $X=0-9 \cdot 10^{8}$ Па при различных значениях $U$. Видно, что обратный ток линейно растет с ростом одноосного сжатия до $X=6 \cdot 10^{8}$ Па, а далее наблюдается более быстрый рост обратного тока. С увеличением приложенного обратного напряжения скорость роста обратного тока при одноосном сжатии увеличивается. Следует отметить, что чувствительность обратного тока структур $\mathrm{Sb}-p-\mathrm{Si}\langle\mathrm{B}, \mathrm{Mn}\rangle-\mathrm{Au}$ к одноосной деформации, в отличие от прямого тока, в широком диапазоне напряжений не перестает быть зависимой от значения приложенного напряжения в обратном направлении.

Для выявления механизма прохождения тока через диодную структуру одноосному упругому сжатию были подвергнуты и образцы компенсированных кристаллов $p$ - $\mathrm{Si}\langle\mathrm{B}, \mathrm{Mn}\rangle$ с исходным удельным сопротивлением $\rho_{0}=3 \cdot 10^{2} \mathrm{OM} \cdot \mathrm{cm}$, из которых была изготовлена база диодов. Проведенные исследования тензопроводимости в образцах $p$ - $\mathrm{Si}\langle\mathrm{B}, \mathrm{Mn}\rangle$ при условиях сжатия $\mathbf{J}\|\mathbf{X}\|[111]$ в интервале $X=0-6 \cdot 10^{8}$ Па при комнатной температуре (рис. 3) показали, что удельное сопротивление компенсированных образцов уменьшает- ся (кривая 2) с ростом величины направленного сжатия $X$. Относительно сильный тензоэффект наблюдается в исходных образцах $p$-Si $\langle\mathrm{B}\rangle$ (кривая 1) с удельным сопротивлением $\rho_{0}=4 \mathrm{OM} \cdot \mathrm{cm}$.

Исследования эффекта Холла, проведенные для выявления механизма тензоэффекта в образцах $p$ - $\mathrm{Si}\langle\mathrm{B}, \mathrm{Mn}\rangle$ при тех же условиях сжатия показали, что тензоэффект в данных образцах обусловлен одновременным изменением (увеличением) концентрации и подвижности

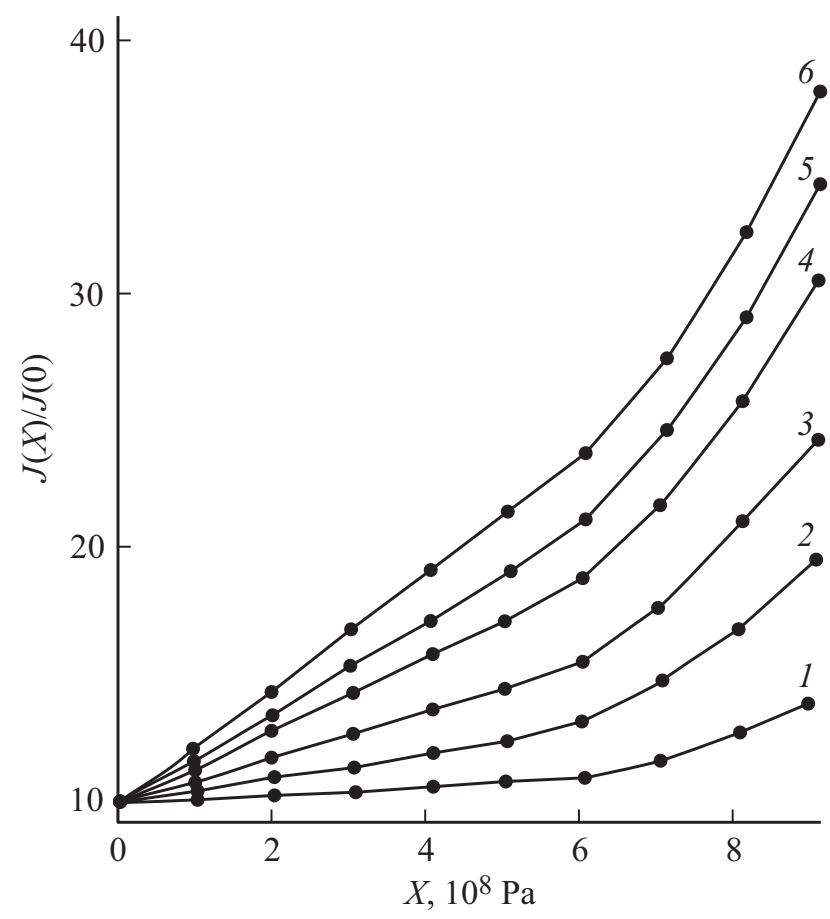

Рис. 2. Зависимости относительного изменения тока обратной ветви ВАХ ПБД $\mathrm{Sb}-p$ - $\mathrm{Si}\langle\mathrm{B}, \mathrm{Mn}\rangle-\mathrm{Au}$ от величины одноосного сжатия. $U=2(1), 6(2), 10(3), 16(4), 20$ (5), 25 В (6).

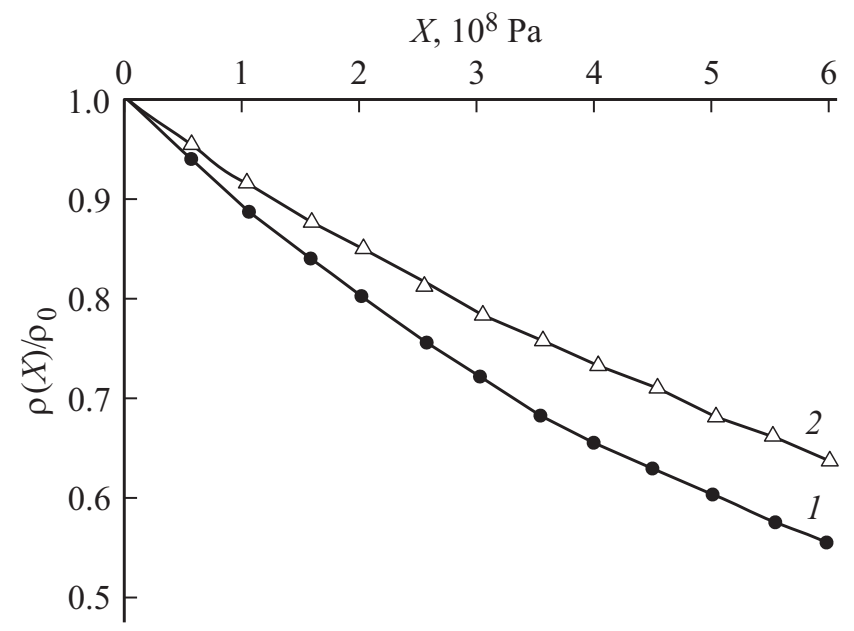

Рис. 3. Тензосопротивление $\rho(X) / \rho_{0}$ образцов при однооосном упругом сжатии и температуре $T=300 \mathrm{~K}$. J $\|\mathbf{X}\|[111]$. Образцы: 1 - исходный $p$-Si $\langle\mathrm{B}\rangle, \quad \rho_{0}=4 \mathrm{OM} \cdot \mathrm{cm} ; 2$ $p$-Si $\langle\mathrm{B}, \mathrm{Mn}\rangle, \rho_{0}=300 \mathrm{OM} \cdot \mathrm{cm}$. 


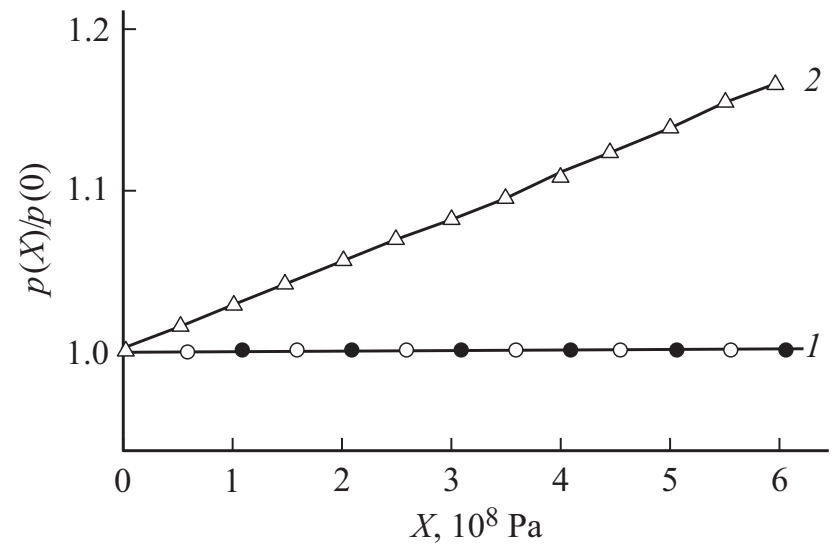

Рис. 4. Зависимости концентрации дырок в образцах при одноосном сжатии: $\mathbf{J}\|\mathbf{X}\|[111]$. Образцы: 1 - исходный $p-\mathrm{Si}\langle\mathrm{B}\rangle, \rho_{0}=4$ Ом $\cdot$ см; $2-p-\mathrm{Si}\langle\mathrm{B}, \mathrm{Mn}\rangle, \rho_{0}=3 \cdot 10^{2}$ Ом $\cdot$ см.

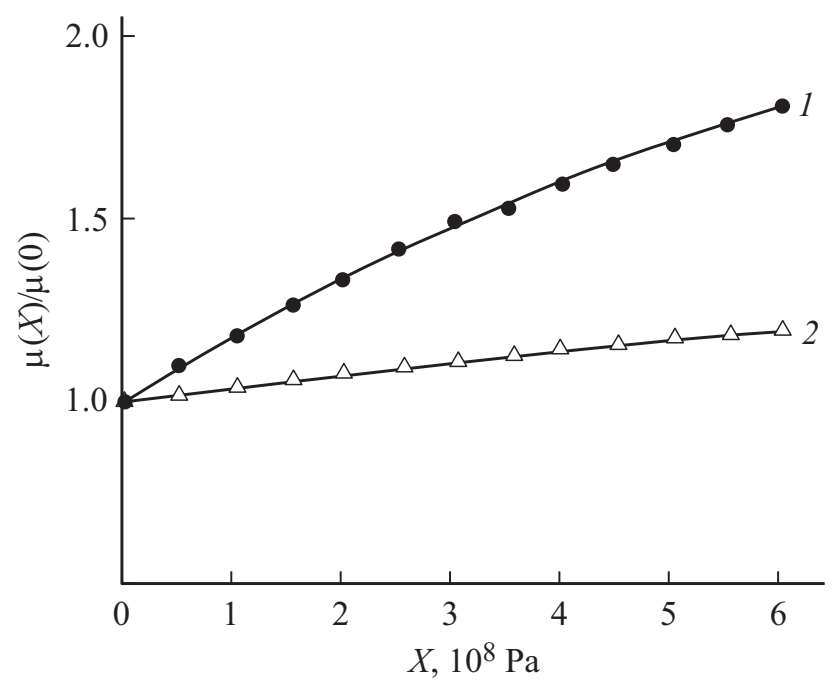

Pис. 5. Зависимости подвижности дырок в образцах при одноосном сжатии: $\mathbf{J}\|\mathbf{X}\|[111]$. Образцы: 1 - исходный $p-\mathrm{Si}\langle\mathrm{B}\rangle, \rho_{0}=4 \mathrm{OM} \cdot \mathrm{cm} ; 2-p-\mathrm{Si}\langle\mathrm{B}, \mathrm{Mn}\rangle, \rho_{0}=3 \cdot 10^{2} \mathrm{OM} \cdot \mathrm{cm}$.

дырок. Концентрация дырок $p(X)$ в образцах $p$-Si $\langle\mathrm{B}\rangle$ во всем диапазоне сжатия остается постоянной (рис. 4 , кривая 1), так как мелкие примесные уровни при комнатной температуре являются полностью опустошенными. В компенсированных образцах $p$ - $\mathrm{Si}\langle\mathrm{B}, \mathrm{Mn}\rangle$ (кривая 2) концентрация дырок увеличивается с ростом $X$. Увеличение концентрации дырок в данных образцах, возможно, связано с изменением степени заполнения глубоких уровней $\mathrm{Mn}$ в $\mathrm{Si}$ за счет изменения их энергии ионизации при одноосном сжатии [2].

Значения подвижности дырок $\mu(X)$ в исходных (рис. 5, кривая 1) и компенсированных образцах $p$ - $\mathrm{Si}\langle\mathrm{B}, \mathrm{Mn}\rangle$ (рис. 5, кривая 2) увеличиваются. Рост подвижности дырок в исходных и слабо компенсированных образцах в условиях симметричного расположения оси деформации относительно всех изоэнергегических эллипсоидов можно связать с расщеплением зоны легких и тяжелых дырок в валентной зоне [3].

Так как при одноосном сжатии подвижность дырок в базе исследуемых диодов с ростом $X$ увеличивается, этим вкладом в изменение тока через диоды нельзя будет пренебрегать. Действительно, согласно [3], изменение тока под давлением выражается как

$$
\frac{J(X)}{J(0)}=\frac{\mu(0)}{\mu(X)} \exp \left(\frac{q \Delta \varphi_{b}}{k T}\right),
$$

где $J(X), \mu(X)$ - значения тока и подвижности дырок при $X \neq 0$. Как видно из $(1)$, при воздействии одноосного сжатия изменение прямого тока ПБД обусловлено встречным изменением подвижности носителей тока в базе и высоты потенциального барьера. Если увеличение подвижности приводит к уменьшению чувствительности, то снижение потенциального барьера приводит к увеличению чувствительности прямого тока к одноосному давлению.

Анализ и сравнение экспериментальных данных показывает, что изменение прямого тока через данный ПБД структуры $\mathrm{Sb}-p$ - $\mathrm{Si}\langle\mathrm{B}, \mathrm{Mn}\rangle-\mathrm{Au}$ нельзя объяснить только изменением подвижности и высоты потенциального барьера. По-видимому, в данном случае значительное увеличение прямого тока ПБД при одноосной упругой деформации происходит благодаря внутренней положительной обратной связи.

Так как база данной диодной структуры частично компенсирована, то при одноосном сжатии за счет увеличения концентрации дырок уменьшается удельное сопротивление базы. Увеличение проводимости базы приводит к перераспределению напряжения между базой и барьером, и происходит внутреннее усиление прямого тока диода.

Увеличение обратного тока ПБД структуры $\mathrm{Sb}-p$ - $\mathrm{Si}\langle\mathrm{B}, \mathrm{Mn}\rangle-\mathrm{Au}$ при одноосном сжатии, по-видимомy, связано с тензогенерацией неравновесных носителей и, следовательно, с увеличением концентрации дырок как в области объемного заряда (ОО3), так и в базе данных диодных структур за счет изменения степени заполнения глубокого уровня Мn. Увеличение токовой чувствительности с повышением приложенного напряжения в обратном направлении можно объяснить увеличением дрейфовой скорости носителей тока в области объемного заряда.

Отметим, что хотя в кремниевых структурах, легированных марганцем, возможно проявление эффектов отрицательного магнитосопротивления, которые могут влиять на определяемые значения концентрации и подвижности носителей, однако это характерно для сильно компенсированных образцов (см., например, [4]). Поскольку в исследованных нами диодных структурах материал базы - слабо компенсированный, удельное сопротивление $\sim 300$ Ом · см, мы полагаем, что превалирующим механизмом уменьшения удельного сопротивления является тензорезистивный эффект. 


\section{4. Заключение}

На основе полученных результатов можно сделать следующие выводы.

- Исследуемые структуры ПБД имеют сравнительно высокую чувствительность по току к одноосному давлению при обратном смещении.

- Увеличение прямого тока данных диодных структур при одноосной упругой деформации обусловлено внутренним усилением, связанным с перераспределением приложенного напряжения между базой и барьером структуры.

- Усиление токовой чувствительности обратно смещенных структур ПБД при одноосном сжатии обусловлено тензогенерацией неравновесных носителей тока в базе и области объемного заряда структуры, а также увеличением их дрейфовой скорости.

\section{Конфликт интересов}

Авторы заявляют, что у них нет конфликта интересов.

\section{Список литературы}

[1] S. Zainabidinov, I.G. Tursunov, O. Khimmatkulov. Semiconductors, 52 (8), 896 (2018).

[2] S. Zainabidinov, O. Mamatkarimov, I.G. Tursunov, O. Khimmatkulov. Ukr. J. Phys., 62 (11), 957 (2017).

[3] А.А. Полякова. Дебормация полупроводников и полупроводниковых приборов (М., Энергия, 1972) гл. 2, с. 34.

[4] М.К. Бахадырханов, К.С. Аюпов, Г.Х. Мавлонов, С.Б. Исамов. ФТП, 44 (9), 1181 (2010).

Редактор Л.В. Шаронова

\section{Effect of uniaxial elastic deformation on current-voltage characteristic of surface-barrier $\mathbf{S b}-\boldsymbol{p}$-Si $\langle\mathbf{M n}\rangle$ diodes}

O.O. Mamatkarimov ${ }^{1}$, O. Khimmatkulov ${ }^{2}$, I.G. Tursunov 3,4

${ }^{1}$ Namangan Institute of Engineering and Technology, 160115 Namangan, Uzbekistan

${ }^{2}$ Taskent State Tecnical University, 100174 Tashkent, Uzbekistan

${ }^{3}$ Chirchik State Pedagogical Institute, 100700 Chichik, Uzbekistan

${ }^{4}$ National University of Uzbekistan, 100174 Tashkent, Uzbekistan 